\title{
Serotonin syndrome induced by the readministration of escitalopram after a short- term interruption in an elderly woman with depression: a case report
}

\author{
This article was published in the following Dove Press journal: \\ Neuropsychiatric Disease and Treatment \\ 30 September 2015 \\ Number of times this article has been viewed
}

\author{
Yasushi Sato ${ }^{1,2}$ \\ Kazuhiko Nakamura' \\ Norio Yasui-Furukori ${ }^{1}$ \\ 'Department of Neuropsychiatry, \\ Graduate School of Medicine, \\ Hirosaki University, Hirosaki, Japan; \\ ${ }^{2}$ Department of Psychiatry, Tsugaru \\ General Hospital, Goshogawara, Japan
}

Background: Serotonin syndrome is a rare but potentially fatal side effect of antidepressants that results from the effects of drug activity on both central and peripheral serotonergic receptors. Case: A 78-year-old Japanese female with a 2-year history of major depressive disorder was treated with escitalopram $(10 \mathrm{mg} / \mathrm{d})$, risperidone $(1 \mathrm{mg} / \mathrm{d})$, and nitrazepam $(5 \mathrm{mg} / \mathrm{d})$. One month after beginning this drug regimen, she was transferred to the emergency department and immediately hospitalized due to suspicion of a urinary tract infection and dehydration. All psychotropic drugs were discontinued. Five days later, the patient's physical condition had recovered; therefore, the same dose of escitalopram $(10 \mathrm{mg} / \mathrm{d})$ was readministered. The patient subsequently developed convulsions accompanied by impaired consciousness, high fever, and myoclonus of both upper extremities. The tendon reflexes of both lower extremities were enhanced. Based on these clinical signs and symptoms, we suspected serotonin syndrome; therefore, escitalopram was discontinued, and a fluid infusion was initiated. The patient recovered from all symptoms within 3 weeks without receiving additional antidepressants.

Conclusion: This case suggests that the careless readministration of selective serotonin reuptake inhibitors (SSRIs) is harmful to at-risk patients, like those in poor physical condition and the elderly.

Keywords: serotonin syndrome, escitalopram, readministration

\section{Introduction}

Serotonin syndrome is a rare but potentially fatal side effect of antidepressants that results from the effects of drug activity on both central and peripheral serotonergic receptors. This syndrome consists of a combination of mental status changes, neuromuscular hyperactivity, and autonomic hyperactivity. ${ }^{1}$ Serotonin syndrome is also associated with increased serotonergic activity in the central nervous system. Selective serotonin reuptake inhibitors (SSRIs) may be the most commonly implicated group of medications associated with serotonin syndrome. ${ }^{1}$

SSRIs are the most common antidepressant drugs used in the treatment of major depressive and anxiety disorders. SSRIs are better tolerated than tricyclic antidepressants (TCAs) by older patients because SSRIs exhibit superior side effect profiles, including fewer anticholinergic side effects. ${ }^{2,3}$ A network meta-analysis suggested that escitalopram is superior to other antidepressants with respect to both efficacy and tolerability. ${ }^{4}$

We present a case of serotonin syndrome induced by the readministration of escitalopram after a short-term interruption in an elderly woman with depression. The
Correspondence: Norio Yasui-Furukori Department of Neuropsychiatry, Hirosaki University, School of Medicine, Hirosaki 036-8562, Japan

Tel $+8 I \quad 172395066$

Fax $+8 I 172395067$

Email yasufuru@hirosaki-u.ac.jp 
Ethics Committee of Hirosaki University, School of Medicine does not require approvals for a case report. Informed consent from the patient to present this clinical course was obtained.

\section{Case}

The patient was a 78-year-old Japanese female with a 2-year history of major depressive disorder, a right putamen infarction 3 years prior to the serotonin syndrome event described in this report and hypertension, an overactive bladder, and depression. She was treated with $10 \mathrm{mg} / \mathrm{d}$ of escitalopram, $30 \mathrm{mg} / \mathrm{d}$ of mirtazapine, and $5 \mathrm{mg} / \mathrm{d}$ of nitrazepam for more than 6 months. At 1 month before the occurrence of serotonin syndrome, mirtazapine was discontinued and replaced with $1 \mathrm{mg} / \mathrm{d}$ of risperidone because the patient had exhibited aggressive behavior toward her husband and had also experienced hallucinations and paranoid delusions. Following this change in her treatment regimen, the patient's psychiatric symptoms improved; however, she developed bradykinesia. Approximately 1 month later, she was transferred to the emergency department of Tsugaru General Hospital due to somnolence, fever, and bradykinesia. A urinalysis was significant for hematuria, a $3+$ test for protein, a $2+$ test for leukocyte esterase, and a specific gravity of at least 1.030. The patient's serum creatinine level, blood urea nitrogen level, white cell count, and C-reactive protein (CRP) level were $0.72 \mathrm{mg} / \mathrm{dL}, 15.4 \mathrm{mg} / \mathrm{dL}, 7,620$ and $0.16 \mu \mathrm{g} / \mathrm{mL}$, respectively. Her liver function indicators and creatine kinase levels were within their normal ranges. The patient was negative for influenza antigens A and B. She was immediately hospitalized in the internal medicine ward of the same hospital due to suspicion of a urinary tract infection and dehydration. Because her physicians believed that her bradykinesia may have been caused by her medications, all psychotropic drugs were discontinued. She was treated with intravenous fluid replacement and levofloxacin $(500 \mathrm{mg} / \mathrm{d})$. Her general condition gradually improved; however, her low-grade fever (from $37.0^{\circ} \mathrm{C}$ to $38.0^{\circ} \mathrm{C}$ ) persisted.

At 5 days following the discontinuation of her psychotropic drugs, treatment with $10 \mathrm{mg} / \mathrm{d}$ of escitalopram was initiated. On the evening of the first day following escitalopram readministration, the patient developed insomnia and confusion and was restrained to ensure her safety. On the morning of the second day following escitalopram readministration, she experienced a convulsive seizure, and her oxygen saturation decreased. Even after the convulsions were suppressed via intravenous diazepam, she remained unconscious (Glasgow Coma Scale: Eye1, Verbal1, and Motor1) and exhibited a high fever (with a maximum fever of $41.5^{\circ} \mathrm{C}$ ) and myoclonus of both upper extremities. Enhanced tendon reflexes of both lower extremities were also observed. The patient did not exhibit muscle rigidity. A urine examination, a blood chemistry test, and a CT scan of the chest and abdomen did not demonstrate the presence of any infectious foci. CT and MRI scans of the brain did not reveal any obvious new lesions. The patient remained negative for influenza antigens A and B. Seizure discharges were not observed on an electroencephalographic examination. Based on these clinical signs and symptoms, we suspected serotonin syndrome. Therefore, escitalopram was discontinued, and a fluid infusion was initiated. All of her symptoms, with the exception of a slight fever, resolved within a few days after the aforementioned studies were obtained. After 3 weeks, the patient had recovered without the administration of additional antidepressants.

\section{Discussion}

The patient exhibited mental status changes, myoclonus, hyperreflexia, and fever after the readministration of escitalopram. Alternative diagnoses were excluded either via history or via laboratory examination, and the observed symptoms met the diagnostic criteria for serotonin syndrome described by Sternbach. ${ }^{5}$ Therefore, based on the patient's clinical course, we concluded that serotonin syndrome had developed due to the readministration of escitalopram. Although no symptoms of serotonin syndrome had been observed during approximately 2 years of treatment with $10 \mathrm{mg} / \mathrm{d}$ of escitalopram, serotonin syndrome developed on the second day following the resumption of treatment with the same dose of escitalopram after a short-term discontinuation.

5-HT receptor activation is a common cause of both serotonin toxicity and serotonin syndrome. ${ }^{6}$ Additionally, blocking 5-HT2A receptors represents a positive response to serotonin syndrome. Cyproheptadine, chlorpromazine, and olanzapine, all of which are capable of blocking 5-HT2A receptors, have exhibited excellent efficacy in the setting of serotonin syndrome. A possible mechanism underlying the development of serotonin syndrome is as follows: firstly, upregulated 5-HT receptors may change following the interruption of escitalopram. Secondly, the plasma concentration of escitalopram may increase following its readministration because of the deterioration of the patient's condition.

Although little data exists regarding the prevalence of escitalopram-induced serotonin syndrome, several cases of this syndrome have been described. One such case involved an 88-year-old female who experienced an interaction between $5 \mathrm{mg}$ of escitalopram and $50 \mathrm{mg}$ of miconazole. ${ }^{7}$ A second case involved a 27 -year-old female who experienced 
an interaction between $10 \mathrm{mg}$ of escitalopram and 50-60 mg of cyclobenzaprine ${ }^{8}$ Finally, a third case involved a 65 -yearold female who experienced an interaction between $10 \mathrm{mg}$ of escitalopram and $600 \mathrm{mg}$ of the antibiotic linezolid. ${ }^{9}$

To the best of our knowledge, no specific clinical characteristics, with the exception of the accidental combination of serotonergic medications, have been associated with a predisposition to serotonin syndrome. In this case, a urinary tract infection and dehydration were observed prior to the development of serotonin syndrome. Therefore, the deterioration of a patient's condition may be a risk factor for serotonin syndrome.

\section{Acknowledgments}

We would like to thank Dr Sakamoto and Dr Sasaki of the Department of Psychiatry of Tsugaru General Hospital for their advice regarding this case.

\section{Disclosure}

Norio Yasui-Furukori has received grant/research support or honoraria from, and been on the speakers of, Asteras, Dainippon, Eli Lilly, GSK, Janssen-Pharma, Meiji Mochida,
MSD, Otsuka, Pfizer, Takada and Yoshitomi. The authors report no other conflicts of interests in this work. The funders had no role in study design, data collection and analysis, decision to publish, or preparation of the manuscript.

\section{References}

1. Volpi-Abadie J, Kaye AM, Kaye AD. Serotonin syndrome. Ochsner J. 2013;13:533-540.

2. Topiwala A, Chouliaras L, Ebmeier KP. Prescribing selective serotonin reuptake inhibitors in older age. Maturitas. 2014;77:118-123.

3. Garnock-Jones KP, McCormack PL. Escitalopram: a review of its use in the management of major depressive disorder in adults. CNS Drugs. 2010;24:769-796.

4. Cipriani A, Furukawa TA, Salanti G, et al. Comparative efficacy and acceptability of 12 new-generation antidepressants: a multiple-treatments meta-analysis. Lancet. 2009;373(9665):746-758.

5. Sternbach H. The serotonin syndrome. Am J Psychiatry. 1991;148: 705-713.

6. Boyer EW, Shannon M. The serotonin syndrome. N Engl J Med. 2005; 352:1112-1120.

7. Baptista G, Eiden C, Monguillot P, Philibert C, Jeandel C. Serotonin syndrome during treatment with low dose of escitalopram associated with miconazole mucoadhesive tablet: a suspected drug interaction. Int Psychogeriatr. 2012;24:845-847.

8. Day LT, Jeanmonod RK. Serotonin syndrome in a patient taking Lexapro and Flexeril: a case report. Am J Emerg Med. 2008;26:1069.e1-e3.

9. Kulkarni RR, Kulkarni PR. Linezolid-induced near-fatal serotonin syndrome during escitalopram therapy: case report and review of literature. Indian J Psychol Med. 2013;35:413-416.
Neuropsychiatric Disease and Treatment

\section{Publish your work in this journal}

Neuropsychiatric Disease and Treatment is an international, peerreviewed journal of clinical therapeutics and pharmacology focusing on concise rapid reporting of clinical or pre-clinical studies on a range of neuropsychiatric and neurological disorders. This journal is indexed on PubMed Central, the 'PsycINFO' database and CAS,

\section{Dovepress}

and is the official journal of The International Neuropsychiatric Association (INA). The manuscript management system is completely online and includes a very quick and fair peer-review system, which is all easy to use. Visit http://www.dovepress.com/testimonials.php to read real quotes from published authors. 